\title{
Polynomial approximation of aerodynamic coefficients based on the statistical description of the wind incidence
}

\author{
V. Denoël* \\ University of Liège, Department of Architecture, Geology, Environment and Construction, Belgium \\ FNRS, National Fund for Scientific Research, Belgium
}

\section{A R T I C L E I N F O}

\section{Article history:}

Received 6 September 2007

Received in revised form

6 May 2008

Accepted 13 May 2008

Available online 21 May 2008

\section{Keywords:}

Aerodynamic coefficient

Non-linear aerodynamics

Wind incidence probability

Quasi-steady model

Stochastic linearization

Bridge aerodynamics

\begin{abstract}
A B S T R A C T
In civil engineering applications, the aerodynamic coefficients are usually measured in wind tunnels for several wind incidences. The measurement results need to be linearized in order to perform the design of the structure. This paper justifies the use of different linearization techniques for different assessments as divergence or buffeting analysis. In this latter context, it is proposed to linearize the aerodynamic coefficient by the least-square method, using the probability density function of the wind incidence as a weighting function. First this probability density function is computed for a 2-D wind flow, as a function of the wind intensities and their correlation. Then, the comparison of results from different linearization techniques provides surprising results indicating that what is usually performed should be considered with care.
\end{abstract}

(c) 2008 Elsevier Ltd. All rights reserved.

\section{Introduction}

Despite recent advances in the numerical simulation of fluidstructure interactions (e.g. [1]), the complete interaction between a turbulent oncoming flow and a bridge deck, spread out along several hundreds of meters, still requires heavy computation efforts. The safety of a bridge deck is therefore usually assessed with respect to a list of phenomena, considered separately. Different wind loading models are used for each assessment [2-4]. Two major families of models are distinguished. The first one provides a precise description of the transient self-excited forces by means of flutter derivatives (Scanlan coefficients). The second family gathers quasi-steady models based on aerodynamic coefficients. These models can eventually allow some kinds of nonlinearities and are used for evaluating static divergence, galloping or buffeting analysis.

Because of the complexity of bridge deck sections, both flutter derivatives and aerodynamic coefficients need to be measured in wind tunnels. Emphasis is generally put on the estimation of the former one only. Indeed flutter derivatives, resulting from a complex dynamic wind-structure coupling,

\footnotetext{
* Corresponding address: University of Liège, Department of Architecture, Geology, Environment and Construction, Division of Mechanics of Solids, Structures and Fluids, B52/3, Room $+1 / 543$, Chemin des Chevreuils, B 4000 Liège 1 , Belgium. Tel.: +32 436693 56; fax: +32 43669192 .

E-mail address: v.denoel@ulg.ac.be.
}

require dedicated identification procedures $[5,6]$. However the aerodynamic coefficients are obtained by a simple measurement of the stationary force applied by the wind flow on the bridge deck, for different values of the wind incidence [7]. The variations of the identified aerodynamic coefficients with respect to the wind incidence are typically non-linear irregular functions. The main scope of this paper is to present how to transform the gross measurement results into a format useable for the design.

At the design stage, the identified Scanlan coefficients are used without any further modification: critical flutter wind velocities are obtained as a direct result of the measured coefficients. On the contrary, the gross results of aerodynamic coefficient measurements are not readily useable for the subsequent assessments. The quasi-steady loading is indeed usually linearized. This requires the aerodynamic coefficients to be linearized too, with respect to the wind incidence. The impact of this linearization was first investigated in the late 1980's [8,9] but was limited to 1-D wind flows. Recently it has been shown, in the context of 2-D and 3-D turbulent flows, that higher order polynomial approximations of significantly non-linear aerodynamic coefficients have to be considered [10]. For these reasons, it is necessary to estimate at least a linear, but also any other polynomial, approximation of aerodynamic coefficients. This paper focuses mainly on providing a linear approximation of the measured coefficients, but the procedure is presented in such a general way that it could be applied to supply any higher order formulation.

The most appropriate linearized form of a measured aerodynamic coefficient may be different from one kind of evaluation 
a

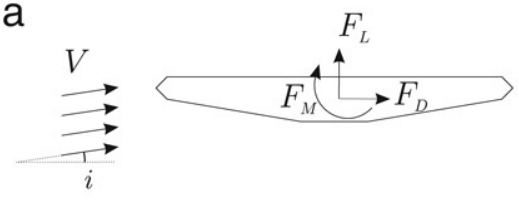

b

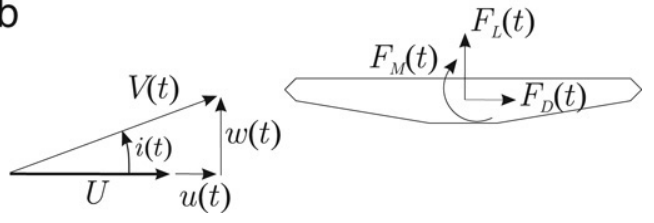

Fig. 1. (a) Aerodynamic forces (drag, lift and moment) on a bridge deck in a uniform laminar flow (b) Extension to a flow with few turbulence, quasi-steady loading.
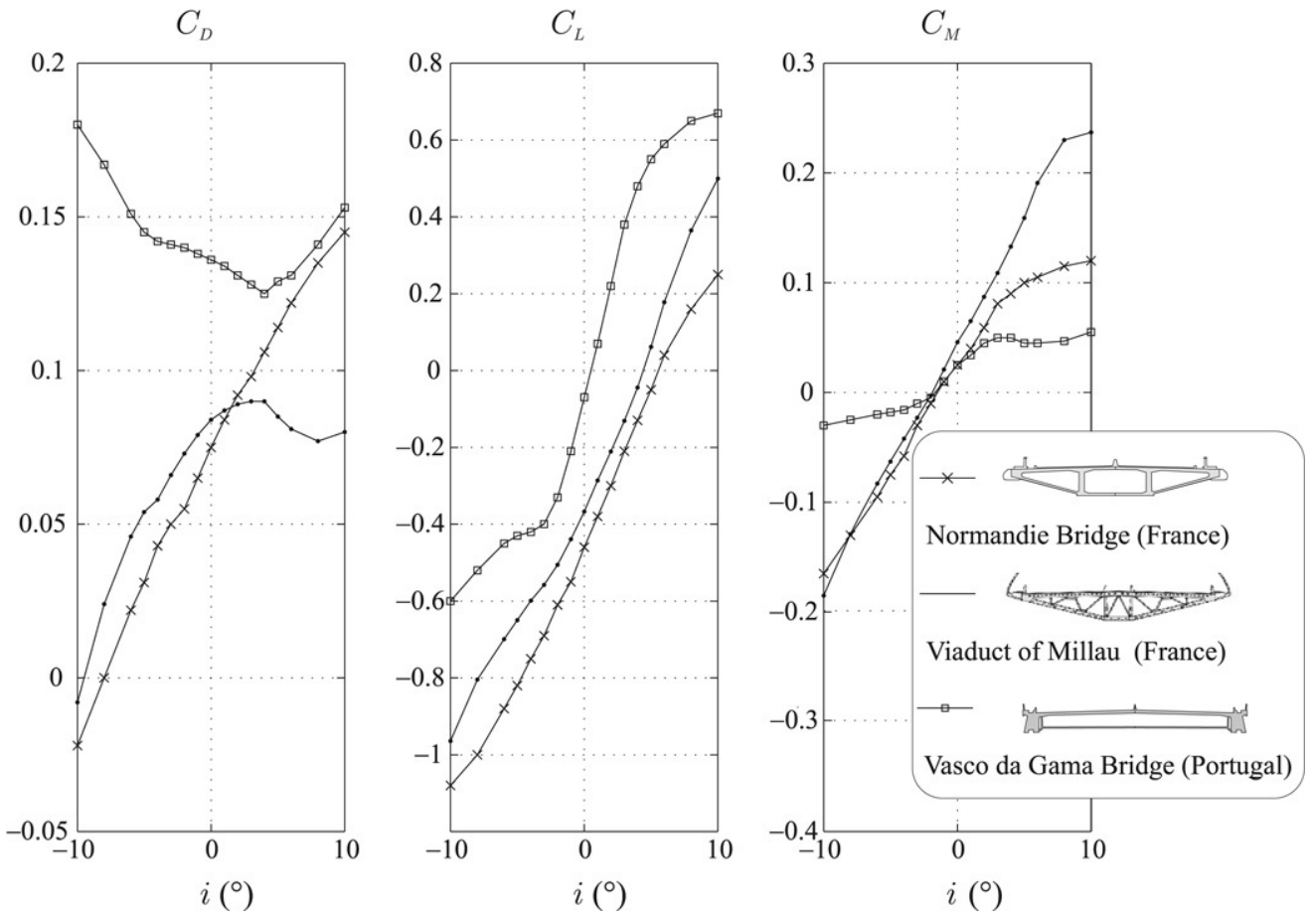

Fig. 2. Examples of aerodynamic coefficients as a function of the wind incidence.

to another. For instance, values around the mean incidence are of major concern when torsional divergence of the bridge deck section (infinitesimal static stability) is considered. In this case a Taylor-McLaurin series expansion around the mean incidence is the best approximation. In the context of a buffeting analysis, the wind incidence changes continuously and it seems logic to weight the measured coefficients by the probability of occurrence of each incidence. A direct application of the stochastic linearization technique [11] results in this conclusion too. It is therefore proposed in this paper to perform the polynomial approximation of the measured aerodynamic coefficients by the least square fitting method with a weighting function proportional to the probability density function (pdf) of the wind incidence.

First, founded on a solid mathematical base, the statistical distribution of the wind incidence is established (2-D wind flow). Since the rigorous expression cannot be used conveniently in practical applications, a simplified version, obtained by a fitting of numerical results, is proposed and its efficiency is demonstrated. The least square fitting of aerodynamic coefficients is then briefly presented in Section 5 with the proposed probability density function used as a weighting function. Finally, the relevance of the proposed technique is demonstrated with a numerical application.

\section{Context, motivations}

The forces acting on a fixed rigid body immersed in a surrounding flow are expressed as (Fig. 1-a):

$F=\frac{1}{2} \rho_{f} C B V^{2}$ where $\rho_{f}$ is the density of the fluid, $C$ is the aerodynamic coefficient (drag, lift or moment), $B$ is a characteristic width and $V$ is the constant fluid velocity. Due to the variability of considered crosssections, the aerodynamic coefficients of bridge deck sections have to be measured in wind tunnels for every new project. Their dependence upon the wind incidence $i$ is generally nonlinear. Fig. 2 depicts some measured aerodynamic coefficients as a function of the wind incidence.

Civil and structural engineering structures are built in the atmospheric boundary layer, which is known to be turbulent. The wind velocity is not constant in time. In 2-D applications, it is thus composed of a mean velocity $U$ and two zero-mean fluctuations $u(t)$ and $w(t)$ (Fig. 1-b). They are usually modelled as Gaussian processes, with a joint probability density function expressed by:

$p_{u w}(u, w)=\frac{1}{2 \pi U^{2} I_{u} I_{w} \sqrt{1-\rho^{2}}} \mathrm{e}^{\frac{-1}{2\left(1-\rho^{2}\right) U^{2}}\left(\frac{u^{2}}{I_{u}^{2}}-\frac{2 \rho u w}{I_{u} I_{w}}+\frac{w^{2}}{I_{w}^{2}}\right)}$

where $I_{u}=\frac{\sigma_{u}}{U}$ and $I_{w}=\frac{\sigma_{w}}{U}$ are the turbulence intensities and $\rho$ is the correlation coefficient between both turbulence components.

As a result, the wind incidence $i(t)$ is also a random process. Its instantaneous value changes continuously (Fig. 1-b) but, in a quasisteady approach, Eq. (1) is still used to express the aerodynamic force applied on the deck. It postulates that the force at a given time depends on the wind components at the same time only, which is valid provided the velocity of the deck is low:

$F(t)=\frac{1}{2} \rho_{f} C[i(t)] B V^{2}(t)$. 


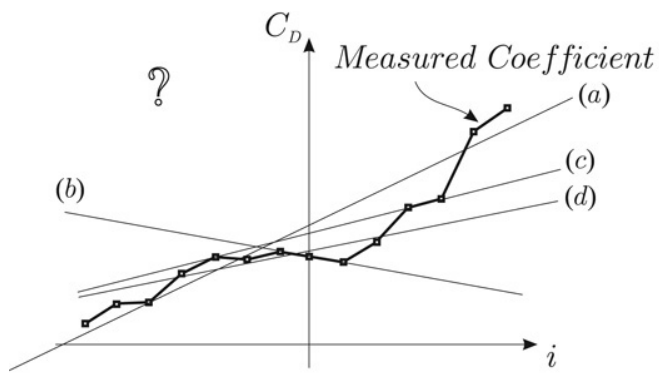

(a)

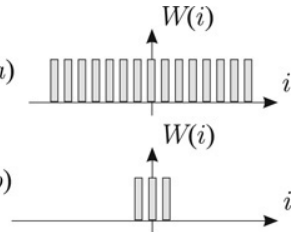

(c)

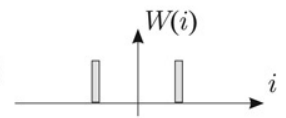

$(d)$

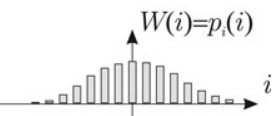

Fig. 3. Schematic illustration of various linearization techniques with the corresponding weighting functions: (a) least-square approximation with uniform weight (b) Taylor-McLaurin series around $i=0$ (c) line passing through two chosen points of the measured function (d) least-square approach, using the pdf of the wind incidence as a weighting function.

The buffeting analysis consists in computing the statistical characteristics of the structural response to this random loading. Because of their non-smoothness, it is not convenient to rigorously handle the measured relation $C[i(t)]$ resulting from the wind tunnel tests. This mathematical difficulty is the inevitable reason for which they have to be replaced by a polynomial approximation.

Usually the aerodynamic coefficients are even linearized (as a function of the wind incidence), in order to simplify the buffeting analysis. A linear approach is indeed very simple because it involves Gaussian processes only. Fig. 3 presents schematically different ways to replace the measurement results by a linear approximation.

First, without any particular bias, the best linear approximation of the measured coefficient is the least square approximation (a). The result is however much dependent on the measurement range. Furthermore this method does not consider the actual wind field.

The Taylor-McLaurin series (b) is known to be the best approximation of a function in the infinitesimal region around a given point. This method should thus be used as long as infinitesimal variations of the wind incidence are concerned (e.g. divergence checking, incipient stability).

In a buffeting analysis, the wind incidence is expected to vary in a finite interval. It is therefore important to fit the aerodynamic coefficient along a finite range. In [12] it is proposed to replace the exact coefficient by a line passing through two intermediate chosen points of the actual measured coefficient (c). These points can be selected in accordance with the range of variation of the wind incidence (e.g. $i_{1}=-I_{w}, i_{2}=+I_{w}$ ). The weighting function corresponding to this method is thus a sum of two Dirac functions, respectively centered at each chosen incidence.

Following the stochastic linearization technique, the best weighting function is the probability density function of the wind incidence (d). Because it captures perfectly the fact that the wind incidence is more often close to its mean value than to extreme ones, this method is the most suitable for buffeting analyses.

The following sections are devoted to the estimation of the pdf of the wind incidence and then to the application of the weighted least square method.

\section{Probability density function of the wind incidence}

The geometric considerations of Fig. 1-b indicate that the wind incidence is expressed as a function of $u$ and $w$ by:

$i(t)=\arctan \frac{w(t)}{U+u(t)}$.

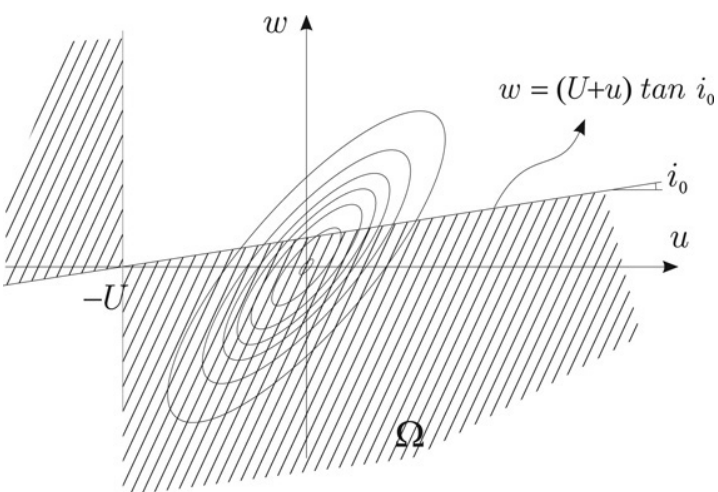

Fig. 4. Subspace of the $(u, w)$ space on which $i<i_{0}$.

The knowledge of the joint pdf of $u$ and $w$, Eq. (1), is strictly necessary for the determination of the cumulative density function (cdf) of $i$ :

$F_{i}\left(i_{0}\right)=\operatorname{prob}\left(i<i_{0}\right)=\operatorname{prob}((u, w) \in \Omega)$

where $\Omega$ is the zone represented by the hatched area in Fig. 4 . In order to keep one-to-one correspondences, it is supposed that $\left.i_{0} \in\right]-\frac{\pi}{2}, \frac{\pi}{2}$ [. The cumulative density function of $i$ is thus obtained by:

$$
\begin{aligned}
& F_{i}\left(i_{0}\right) \\
& \quad=\left(\int_{-\infty}^{-U} \int_{(U+u) \tan i_{0}}^{+\infty}+\int_{-U}^{+\infty} \int_{-\infty}^{(U+u) \tan i_{0}}\right) p_{u w}(u, w) \mathrm{d} w \mathrm{~d} u .
\end{aligned}
$$

The probability density function of $i$ is obtained by a differentiation of Eq. (5) with respect to $i_{0}$. Thanks to the continuity of $p_{u w}(u, w)$, it is expressed by:

$p_{i}(i)=\left(\int_{-U}^{+\infty}-\int_{-\infty}^{-U}\right) p_{u w}(u,(U+u) \tan i) \frac{U+u}{\cos ^{2} i} \mathrm{~d} u$.

Because the considered turbulence intensities are supposed to be small $\left(I_{u}<20 \%, I_{w}<20 \%\right)$, the integrand is very small for $u<-U$. The second domain of integration is therefore neglected and the lower boundary of the first one is extended from $-U$ to $-\infty$ :

$\tilde{p}_{i}(i)=\int_{-\infty}^{+\infty} p_{u w}(u,(U+u) \tan i) \frac{U+u}{\cos ^{2} i} \mathrm{~d} u$

This new relation is not really a rigorous probability density function because it does not fulfil the first Kolmogorov-Smirnov 
a

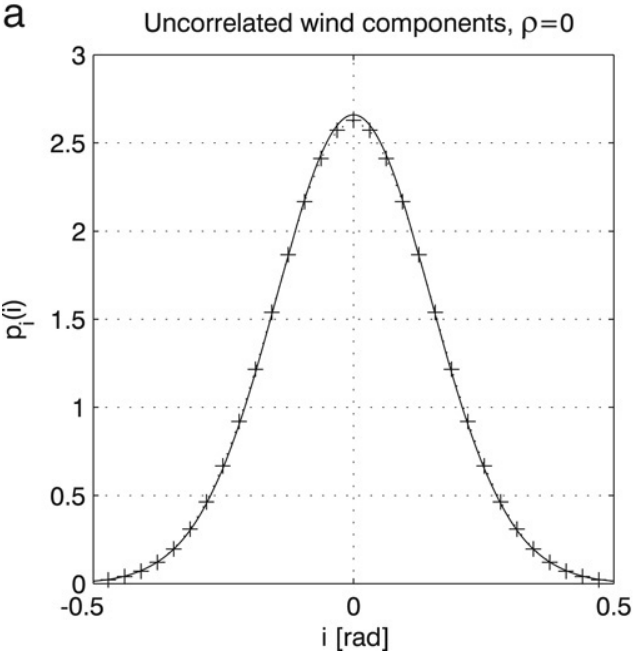

b

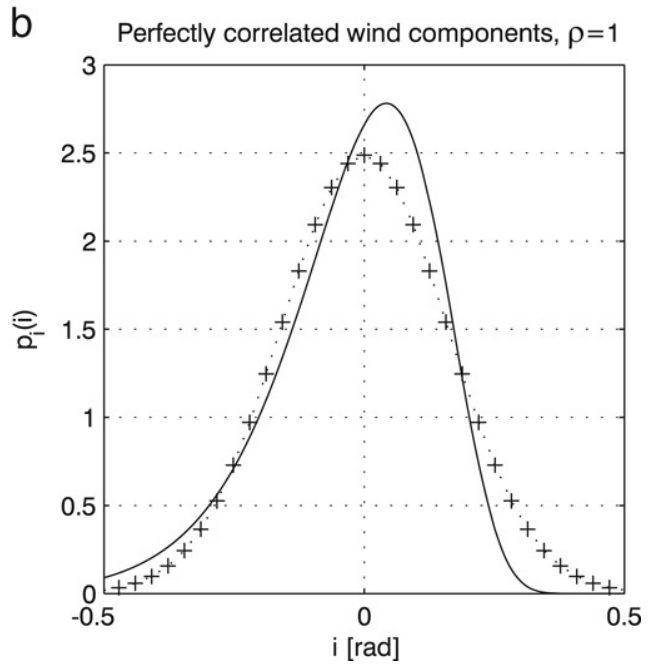

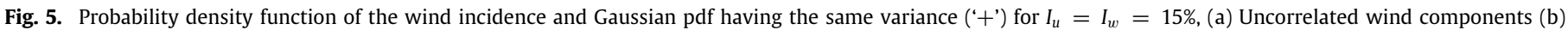
Perfectly correlated wind components.

axiom anymore. However, it can be numerically checked that:

$$
\begin{aligned}
& \left|\int_{-\frac{\pi}{2}}^{+\frac{\pi}{2}} p_{i}(i) i^{k} \mathrm{~d} i-\int_{-\frac{\pi}{2}}^{+\frac{\pi}{2}} \tilde{p}_{i}(i) i^{k} \mathrm{~d} i\right|<10^{-4} \\
& \quad \forall\left(I_{u}, I_{w}\right) \in[0 ; 0.2] \times[0 ; 0.2]
\end{aligned}
$$

for $k=0,1, \ldots 4$. If $\rho$ is kept as a parameter, the introduction of the joint pdf of $u$ and $w$ into Eq. (7) results in tedious computations and leads to practically unusable results. The integral appearing in Eq. (7) has to be performed in a numerical way. This is presented in Section 3.3. Since analytical developments are interesting, at least for the validation of the numerical procedure, two extreme situations are however further investigated in an analytical way. They are presented in the following sections.

\subsection{Uncorrelated wind components $(\rho=0)$}

When the cross-correlation between wind components is not given, they are sometimes supposed to be uncorrelated ( $\rho=$ 0 ). In this case, the introduction of (2) into (7) leads, after some developments, to:

$\tilde{p}_{i}(i)=\frac{I_{w}^{2} \mathrm{e}^{\frac{-1}{2} \frac{\tan ^{2} i}{I_{w}^{2}+I_{u}^{2} \tan ^{2} i}}}{\sqrt{2 \pi} \cos ^{2} i\left(I_{w}^{2}+I_{u}^{2} \tan ^{2} i\right)^{3 / 2}}$.

As an example, this relation is represented in Fig. 5-a for $I_{u}=$ $I_{w}=15 \%$. In this figure, the gaussian distribution having the same variance is also reproduced. The coincidence of both results is remarkable. In spite of its complexity, Eq. (9) presents however the advantage to show first that the actual pdf of the wind incidence is not exactly Gaussian. Furthermore it indicates that the pdf is an even function: $\widetilde{p}_{i}(i)=\widetilde{p}_{i}(-i)$. All odd statistical moments are thus equal to zero:

$\int_{-\frac{\pi}{2}}^{+\frac{\pi}{2}} i^{2 k+1} \widetilde{p}_{i}(i) \mathrm{d} i$

which is expected because the only dissymmetry in $i$ could come either from a non-symmetric $w$ process (which is not the case), either from a non-zero correlation between $u$ and $w$. As a particular case $(k=0)$, the wind incidence is a zero-mean process.

Because the exact expression of the pdf is too complex, it could be interesting to determine an equivalent Gaussian distribution, simply defined as having the same variance. The variance of the wind incidence is obtained by:

$\sigma_{i}^{2}=\int_{-\frac{\pi}{2}}^{+\frac{\pi}{2}} \tilde{p}_{i}(i) i^{2} \mathrm{~d} i=2 \int_{0}^{+\frac{\pi}{2}} \tilde{p}_{i}(i) i^{2} \mathrm{~d} i$

which is simplified by introducing $x=\frac{\tan ^{2} i}{I_{w}^{2}+I_{u}^{2} \tan ^{2} i}$ :

$\sigma_{i}^{2}=\frac{1}{\sqrt{2 \pi}} \int_{0}^{1 / I_{u}^{2}} \frac{\mathrm{e}^{\frac{-x}{2}}}{\sqrt{x}} \arctan ^{2} \sqrt{\frac{I_{w}^{2} x}{1-I_{u}^{2} x}} \mathrm{~d} x$.

If the arctan function is replaced by its Taylor series expansion around $x=0$, a simple expression is obtained for the variance of the incidence:

$\sigma_{i}^{2} \simeq I_{w}^{2} \underbrace{\frac{1}{\sqrt{2 \pi}} \int_{0}^{1 / I_{u}^{2}} \sqrt{x} \mathrm{e}^{\frac{-x}{2}} \mathrm{~d} x}_{\varphi^{2}\left(I_{u}\right)}$.

For small longitudinal turbulence intensities $\left(I_{u}<20 \%\right)$, the upper limit of the integral can be changed to $+\infty$. This provides a Poisson integral $\left(\varphi^{2}=1\right)$ and the expression is simplified to:

$\sigma_{i} \simeq I_{w}$.

This simple relation may be useful for a simple but rough design. A more precise expression, resulting from the numerical integration procedure, is proposed in Section 3.3.

\subsection{Perfectly correlated wind components $(\rho= \pm 1)$}

As a second particular case, it is interesting to consider perfectly correlated wind components. Some authors consider that the origin of both turbulent components lies in the formation of the same eddies and results therefore in a high correlation. In this paper, an in-phase correlation only is considered $(\rho=+1)$ but similar developments can be derived for a negative correlation. For perfectly correlated wind components,

$\frac{u}{\sigma_{u}}=\frac{w}{\sigma_{w}}$.

Because of this perfect correlation, the wind incidence is now expressed as a simple function of $u$ :

$i=\arctan \frac{w}{U+u}=\arctan \frac{I_{w}}{I_{u}} \frac{u}{U+u}$. 


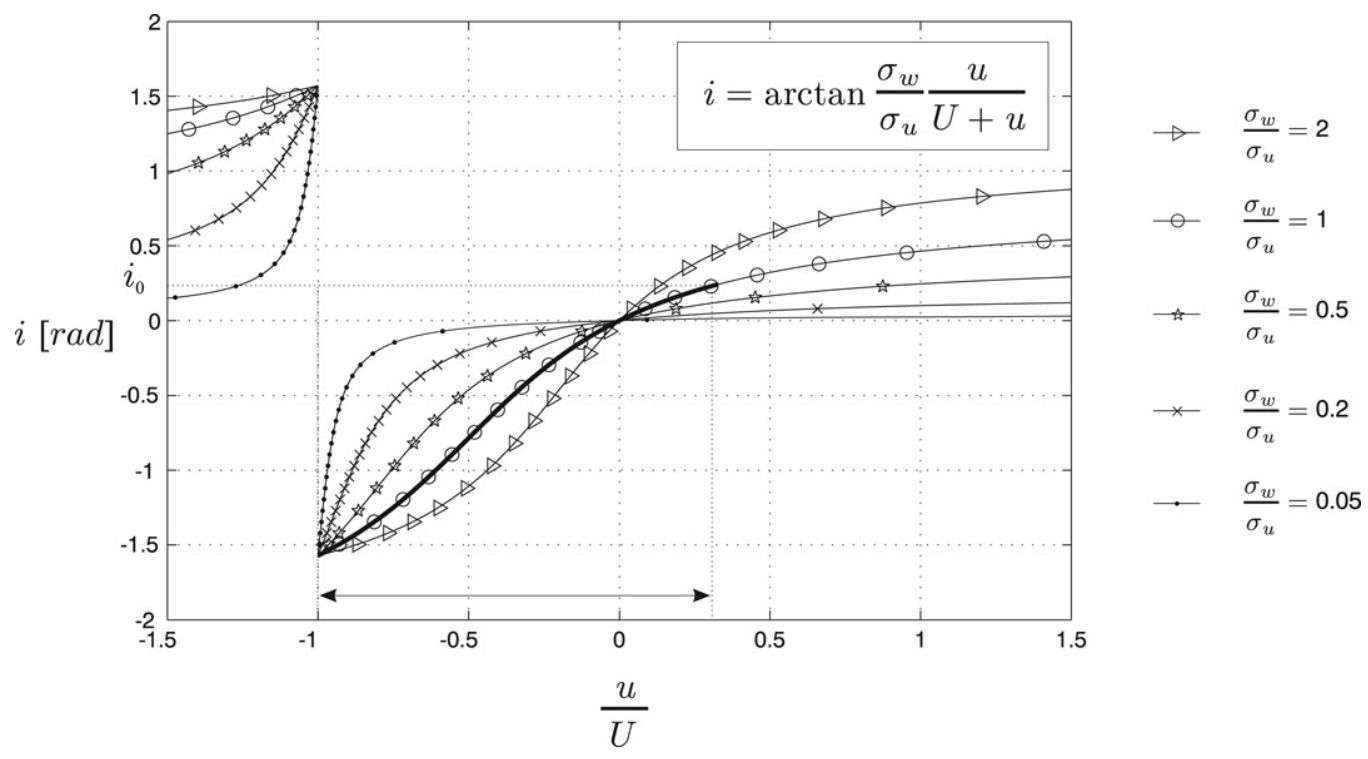

Fig. 6. Relation between the wind incidence and the longitudinal component (perfectly correlated turbulence).

This relation is represented in Fig. 6 for various ratios of turbulence intensities. It has a horizontal asymptote $i_{L}=\arctan \frac{I_{w}}{I_{u}}$ but, since $u / U$ is supposed to remain small, it makes actually no sense to observe incidences larger than $i_{L}$ (values of $u$ smaller than $U$ ). Since $u(i)$ is not a continuous function (see Fig. 6 , care should be taken when writing the cumulative density function of $i$ :

$F_{i}\left(i_{0}\right)=\operatorname{prob}\left(i<i_{0}\right)=\left\{\begin{array}{l}\int_{-U}^{u\left(i_{0}\right)} p_{u}(u) \mathrm{d} u \text { for } i_{0}<i_{L} \\ 1-\int_{u\left(i_{0}\right)}^{-U} p_{u}(u) \mathrm{d} u \text { for } i_{0}>i_{L} .\end{array}\right.$

The probability density function of $i$ results from a side-by-side differentiation of Eq. (17):

$p_{i}(i)=p_{u}[u(i)] \frac{\mathrm{d} u}{\mathrm{~d} i}=\frac{I_{w} \mathrm{e}^{\frac{-1}{2} \frac{\tan ^{2} i}{\left(I_{w}-I_{u} \tan i\right)^{2}}}}{\sqrt{2 \pi} \cos ^{2} i\left(I_{w}-I_{u} \tan i\right)^{2}} \quad \forall i \neq i_{L}$.

This relation is represented in Fig. 5-b for $I_{u}=I_{w}=15 \%$, along with the Gaussian distribution having the same variance. Because of the correlation between both turbulence components, the wind incidence is now significantly skewed. A Gaussian approximation is no longer valid. Higher statistical moments are needed for a better representation of the statistical distribution. These moments are defined by:

$m_{k}=\int_{-\frac{\pi}{2}}^{+\frac{\pi}{2}} i^{k} p_{i}(i) \mathrm{d} i=\int_{-\infty}^{+\infty}[i(u)]^{k} p_{u}(u) \mathrm{d} u$.

Again, the computation of these definite integrals (the first one as well as the second one) provides results of no practical use if the rigorous expression of $p_{i}(i)$ is kept. However the replacement of $[i(u)]$ by its Taylor series expansion around $\frac{u}{U}=$ 0 , provides convenient expressions for the statistical moments. Instead of these, a set of four other quantities, referred to as statistical characteristics in the following, having a simpler physical interpretation are used. The mean $\left(\mu_{i}\right)$, standard deviation $\left(\sigma_{i}\right)$, skewness coefficient $\left(\gamma_{3, i}\right)$ and excess coefficient $\left(\gamma_{e, i}\right)$ are obtained from the statistical moments by:

$\mu_{i}=m_{1}$

$\sigma_{i}=\sqrt{m_{2}-m_{1}^{2}}$ $\gamma_{3, i}=\frac{m_{3}-3 m_{1} m_{2}+2 m_{1}^{3}}{\sigma_{i}^{3}}$

$\gamma_{e, i}=\frac{m_{4}-4 m_{1} m_{3}+6 m_{1}^{2} m_{2}-3 m_{1}^{4}}{\sigma_{i}^{4}}-3$.

It has to be noted that terms until the 6 th order in the series expansion of $[i(u)]$ have to be kept for an accurate estimation of the skewness and excess coefficients. The resulting statistical characteristics, truncated to the second relative order, are:

$\mu_{i}=-I_{u} I_{w}\left(1+3 I_{u}^{2}-3 I_{w}^{2}\right)$

$\sigma_{i}=I_{w}\left(1+4 I_{u}^{2}\right)$

$\gamma_{3, i}=-I_{u}\left(6-42 I_{w}^{2}+44 I_{u}^{2}\right)$

$\gamma_{e, i}=8\left(9 I_{u}^{2}-I_{w}^{2}\right)+16\left(81_{u}^{4}-94_{w}^{2} I_{u}^{2}+4 I_{w}^{4}\right)$.

Compared to the uncorrelated case, the first two characteristics are almost unchanged. The non-Gaussianity is however now a matter of fact and is quantified by significant 3rd and 4th order coefficients. These simplified expressions indicate that the standard deviation of the wind incidence is governed by the transverse turbulence $I_{w}$, whereas its skewness depends essentially on the longitudinal one $I_{u}$.

\subsection{Simplified expressions for any correlation}

In some applications, precise values of the correlation between both turbulence components are accessible. This is the case if cross-power spectral densities are considered at the design stage [13] or if wind tunnels measurements with simulation of the boundary layer are performed. In this case, the synchronous measurement of wind velocity histories in both directions provides the required correlation.

As in the previous sections, it is desired to formulate the statistical moments of the wind incidence as simple functions of $I_{u}, I_{w}$ and $\rho$. The same analytical developments as those performed in the previous sections are not possible. For example a Taylor series expansion of the integrand in Eq. (7) for small $I_{u}$ and $I_{w}$ is not realizable because $p_{u w}$ degenerates then into a 2-D Dirac function. Because no analytical solution is able to provide the desired simplified relations, a numerical procedure is adopted. 

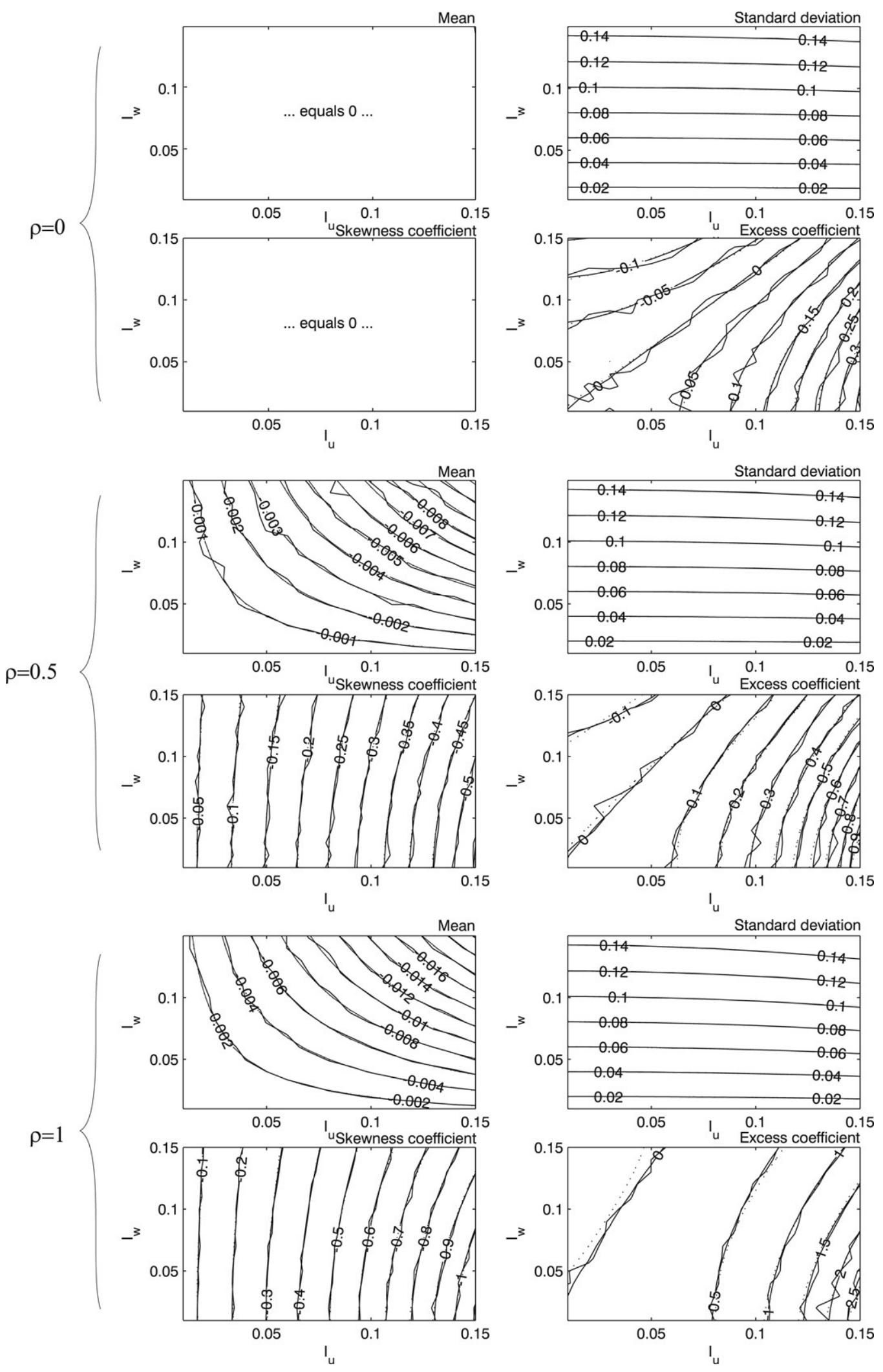

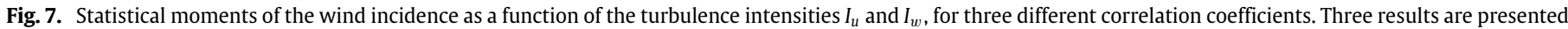
in each graph: numerical integration of the pdf (smooth solid labelled lines), Monte Carlo simulation (solid erratic lines), polynomial fitting (dotted lines).

\subsubsection{Numerical computation of the statistical moments}

For a given triplet $\left(I_{u}, I_{w}, \rho\right)$, the probability density function of the wind incidence Eq. (7) is computed point-by-point for 1500 values uniformly distributed on $\left[\frac{-\pi}{2} ; \frac{\pi}{2}\right]$. For each of them, the numerical integration is performed with the trapezoidal rule for 2000 values of $u$ uniformly distributed on $[-3 U ; 3 U]$. From this numerical estimation of the pdf, the statistical moments of $i$ are computed by a second numerical integration, as defined in Eq. (19). The statistical characteristics corresponding to the given triplet 
Table 1

Coefficients of the polynomial form (Eq. (22)) for each statistical characteristic

\begin{tabular}{|c|c|c|c|c|}
\hline & Mean $\mu_{i}$ & St. dev. $\sigma_{i}$ & Skewness $\gamma_{3, i}$ & Excess $\gamma_{e, i}$ \\
\hline$P_{u}(\rho)$ & 0 & 0 & $-5.8 \rho$ & $-1.4 \rho^{4}+0.24 \rho^{2}+0.086$ \\
\hline$P_{w}(\rho)$ & 0 & 1 & $-0.16 \rho$ & $0.81 \rho^{4}+0.32 \rho^{2}-0.0025$ \\
\hline$P_{u^{2}}(\rho)$ & $0.06 \rho$ & $-0.0082 \rho^{2}-0.0042$ & $-3.4 \rho^{3}+3.4 \rho$ & $29 \rho^{4}-0.14 \rho^{2}+6.2$ \\
\hline$P_{u w}(\rho)$ & $-\rho$ & $0.16 \rho^{2}+0.027$ & $-1.9 \rho^{3}-4.6 \rho$ & $-6 \rho^{4}+71 \rho^{2}+5.4$ \\
\hline$P_{w^{2}}(\rho)$ & $-0.047 \rho$ & $0.034 \rho^{2}-0.0046$ & $2.1 \rho^{3}+2.5 \rho$ & $-7.5 \rho^{4}-22 \rho^{2}-10$ \\
\hline$P_{u^{3}}(\rho)$ & $-0.28 \rho$ & $0.51 \rho^{2}+0.21$ & $9.3 \rho^{3}-85 \rho$ & $-147 \rho^{4}+748 \rho^{2}+61$ \\
\hline$P_{u^{2} w}(\rho)$ & $-0.59 \rho$ & $2.2 \rho^{2}+1.6$ & $37 \rho^{3}+47 \rho$ & $-120 \rho^{4}-704 \rho^{2}-46$ \\
\hline$P_{u w^{2}}(\rho)$ & $0.81 \rho$ & $-1.3 \rho^{2}-0.33$ & $-8.4 \rho^{3}+41 \rho$ & $115 \rho^{4}-107 \rho^{2}-19$ \\
\hline$P_{w^{3}}(\rho)$ & $0.17 \rho$ & -0.85 & $-6.6 \rho^{3}-12 \rho$ & $8 \rho^{4}+98 \rho^{2}+20$ \\
\hline
\end{tabular}

are finally obtained as in Eq. (20). This computation is repeated for 9 different values of the correlation coefficient (from $\rho=$ -1 to $\rho=+1)$, and for $15 \times 15$ couples $\left(I_{u}, I_{w}\right)$. The contour plots of the statistical characteristics are represented by solid lines in Fig. 7. Results are displayed for three correlation coefficients only. They illustrate the main tendencies of the previous analytical developments.

In case of uncorrelated wind components $(\rho=0)$, the mean and skewness coefficient of the wind incidence are equal to zero. Also, the standard deviation is approximately equal to the transverse intensity $I_{w}$, as expressed by Eq. (14). The excess coefficient is not equal to zero, however, which indicates that the wind incidence is not rigorously a Gaussian process.

In case of perfectly correlated wind components $(\rho=1)$, the mean is a hyperbolic function of $I_{u}$ and $I_{w}$. The standard deviation is again almost equal to the transverse intensity $I_{w}$. Fig. 7 indicates also that the skewness is almost equal to six times the longitudinal intensity $I_{u}$, as expressed by Eq. (21).

\subsubsection{Monte Carlo simulation}

In order to validate this numerical procedure as well as the mathematical developments and simplifications mentioned in Eq. (4) to (7), a Monte Carlo simulation is applied. For each considered triplet $\left(I_{u}, I_{w}, \rho\right)$, appropriate series of $5.10^{5}$ values are generated for $u$ and $w$. A series of wind incidences is then built up, by considering Eq. (4). From this series, the statistical characteristics of the wind incidence are computed. They are represented in Fig. 7 by erratic solid lines. The unsmoothness could be rubbed out by considering longer series but these results are sufficient to indicate that the main tendencies are similar to those obtained with the numerical developments. This demonstrates not only the validity of the numerical procedure but also the legitimacy of the simplifications performed in Eq. (4) to (7).

\subsubsection{Polynomial approximation}

In order to propose simple relations for the estimation of the statistical characteristics, 2-D polynomial fittings of the results obtained by the numerical procedure are performed. For each considered correlation coefficient and each statistical characteristic ( $f=\mu_{i}, \sigma_{i}, \gamma_{3, i}$ or $\gamma_{e, i}$ ), the numerical procedure provides a set of $15 \times 15$ values corresponding to various turbulence intensities. They are approximated by:

$$
\begin{aligned}
f & \left(I_{u}, I_{w}, \rho\right)=\underbrace{P_{u}(\rho) I_{u}+P_{w}(\rho) I_{w}}_{01} \\
& +\underbrace{P_{u^{2}}(\rho) I_{u}^{2}+P_{u w}(\rho) I_{u} I_{w}+P_{w^{2}}(\rho) I_{w}^{2}}_{02} \\
& +\underbrace{P_{u^{3}}(\rho) I_{u}^{3}+P_{u^{2} w}(\rho) I_{u}^{2} I_{w}+P_{u w^{2}}(\rho) I_{u} I_{w}^{2}+P_{w^{3}}(\rho) I_{w}^{3}}_{03}
\end{aligned}
$$

where the coefficients $P_{u}(\rho), P_{w}(\rho), \ldots P_{w^{3}}(\rho)$ are computed in such a way to minimize the squared difference between this function and the set of $15 \times 15$ values (least square fitting). As suggested by the contour plots in Fig. 7, a third order approximation is probably more than necessary for the standard deviation, but needed, however, for an accurate representation of the skewness and excess coefficients.

The optimum coefficients are represented in Fig. 8. They are sorted out by order and statistical characteristic. The smoothness of these functions of $\rho$ bears witness to the regularity of the results provided by the numerical procedure and the former least square fitting.

The necessity to use a third order polynomial approximation can be judged by the relative scales obtained for the different orders.

As a final step, polynomial fittings corresponding to these functions of $\rho$ have to be given. The proposed expressions are listed in Table 1. Together with Eq. (22), they provide a simple and convenient way to estimate the statistical characteristics of the wind incidence for any triplet $\left(I_{u}, I_{w}, \rho\right)$. In Fig. 7, the dotted lines represent the contour plots obtained with this simplified method. The good agreement with both other results shows the precision of the proposed relations.

\section{Recomposition of the probability distribution of $i$}

A convenient way to represent the probability density function of a non-Gaussian variable, of which the first four statistical characteristics are given, is the Egdeworth series expansion [14]:

$$
\begin{aligned}
\widehat{p_{i}}(i)= & \Psi(i)\left[1+\frac{\gamma_{3, i}}{6} H_{3}\left(\frac{i-\mu_{i}}{\sigma_{i}}\right)+\frac{\gamma_{e, i}}{24} H_{4}\left(\frac{i-\mu_{i}}{\sigma_{i}}\right)\right. \\
& \left.+\frac{\gamma_{3, i}^{2}}{72} H_{6}\left(\frac{i-\mu_{i}}{\sigma_{i}}\right)\right]
\end{aligned}
$$

This relation expresses that the Gaussian probability density function $\Psi(i)=\frac{1}{\sqrt{2 \pi} \sigma_{i}} \exp \left[\frac{-1}{2}\left(\frac{i-\mu_{i}}{\sigma_{i}}\right)^{2}\right]$ has to be modified by a factor accounting for the non-Gaussianity, i.e. higher statistical characteristics. The Hermite polynomials $\mathrm{H}_{3}, \mathrm{H}_{4}$ and $\mathrm{H}_{6}$ are:

$$
\begin{aligned}
& H_{3}(x)=x^{3}-3 x \\
& H_{4}(x)=x^{4}-6 x^{2}+3 \\
& H_{6}(x)=x^{6}-15 x^{4}+45 x^{2}-15 .
\end{aligned}
$$

The major drawback of this asymptotic probability description is that it can take negative values for very large skewness and/or kurtosis. The non-positiveness is not significant for the considered application. Indeed, Fig. 7 shows that the skewness and excess coefficients are respectively limited to small values $\gamma_{3, i} \simeq 1$ and $\gamma_{e, i} \simeq 2$.

The approximate pdf of the wind incidence given by Eq. (23) is used in the following developments as a weighting function for the least square fitting. 

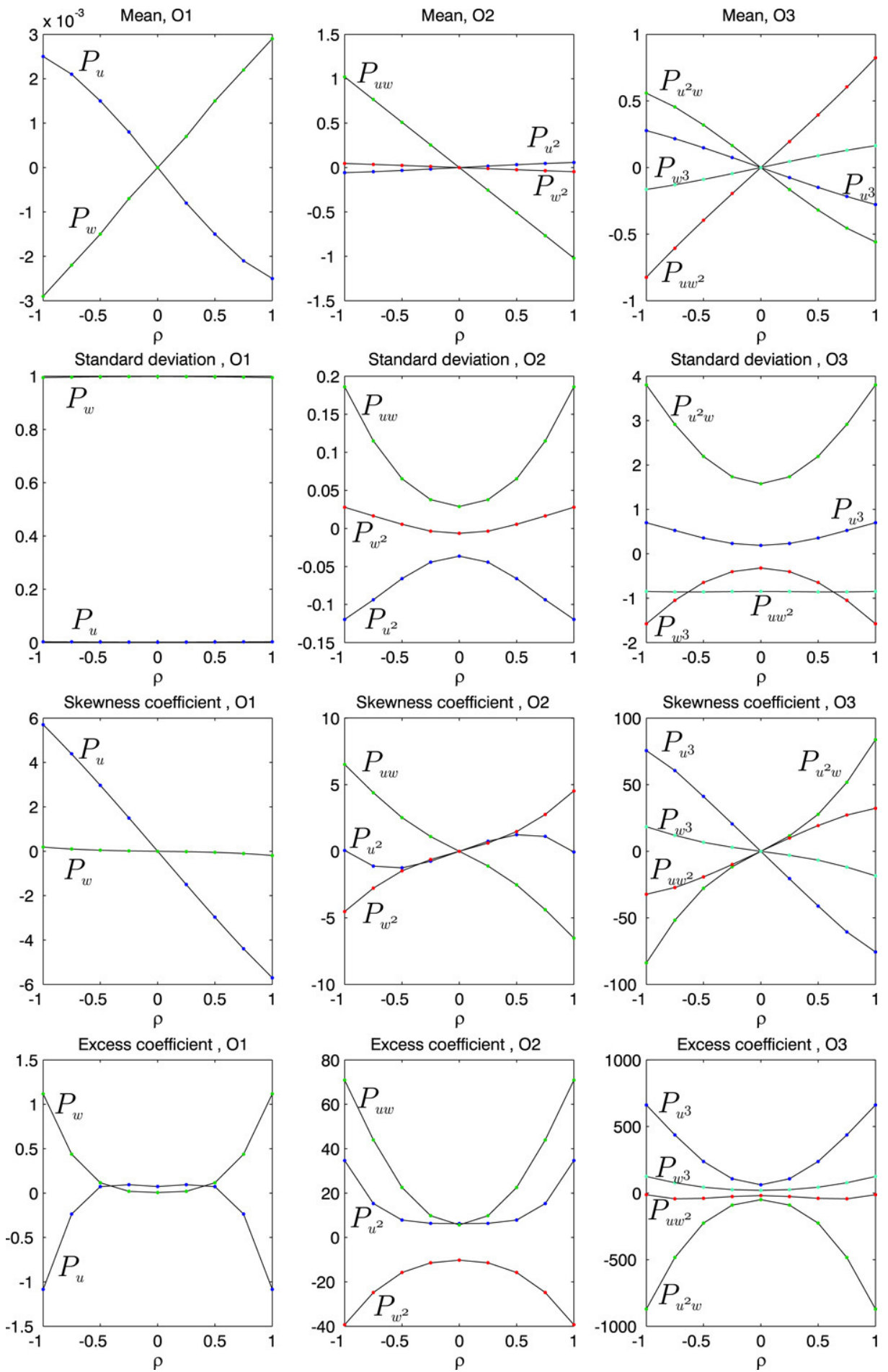

Fig. 8. Coefficients of the polynomial approximation of statistical characteristics (expressed as a function of the correlation between both wind components).

\section{Least square fitting of aerodynamic coefficients}

The measurement of an aerodynamic coefficient through wind tunnel experiments results in a discrete representation:

$\left(i_{k}, C_{k}\right) \quad k=1, \ldots, N$

where $C_{k}$ represents the value measured for incidence $i_{k}$. It is desired to find the least square polynomial fitting of these data with a given weighting function Eq. (23). The $r$ th order polynomial to be fitted is expressed by:

$q^{(r)}(x)=\sum_{m=1}^{r+1} \alpha_{m} x^{m-1}$

where $\{\alpha\}=\left\langle\alpha_{1}, \ldots, \alpha_{r+1}\right\rangle^{\mathrm{T}}$ is the vector of the coefficients to be fitted. Conformingly to the least square method, the function to minimize is:

$\epsilon\left(\alpha_{1}, \ldots, \alpha_{r+1}\right)=\sum_{k=1}^{N} \widehat{p_{i}}\left(i_{k}\right)\left[C_{k}-q^{(r)}\left(i_{k}\right)\right]^{2}$ 

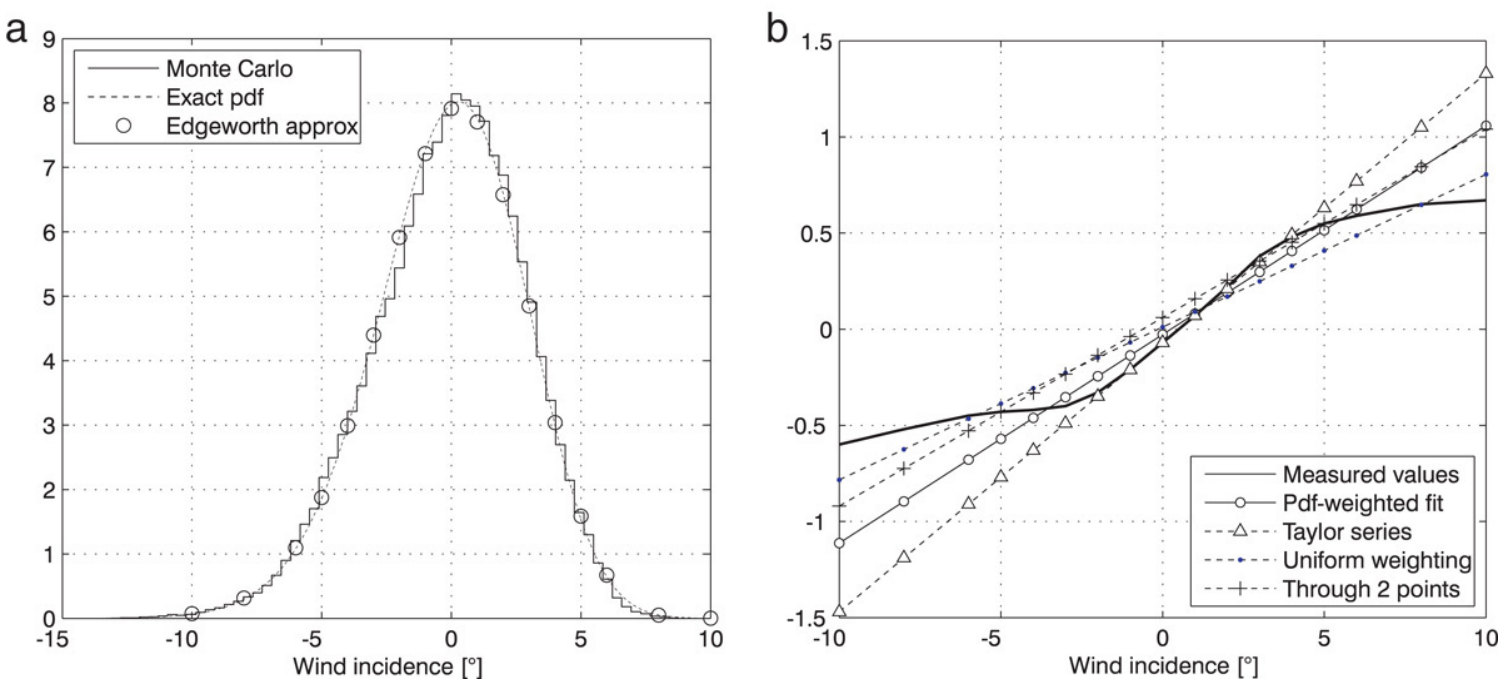

Fig. 9. (a) Probability density function of the wind incidence $\left(I_{u}=I_{w}=5 \%, \rho=1\right)$ (b) Linearization of the lift coefficient of the Vasco da Gama bridge deck

Table 2

Statistical characteristics of the wind incidence obtained with several methods

\begin{tabular}{lllll}
\hline & $\mu_{i}$ & $\sigma_{i}$ & $\gamma_{3, i}$ & $\gamma_{e, i}$ \\
\hline Proposed method & -0.0024 & 0.0508 & -0.300 & 0.156 \\
Monte Carlo & -0.0023 & 0.0505 & -0.301 & 0.151 \\
Theroretical, from Eq. (7) & -0.0025 & 0.0504 & -0.297 & 0.156 \\
\hline
\end{tabular}

The optimum coefficients $\{\alpha\}$ are obtained by the resolution of the so-called normal equations [15]:

$[B][A]\{\alpha\}=[B]\{C\}$

where $A_{k m}=i_{k}^{m-1}, B_{m k}=\widehat{p}_{i}\left(i_{k}\right) A_{k m}(k=1 \ldots N, m=1 \ldots r+1)$ and $\{C\}=\left\langle C_{1}, \ldots, C_{N}\right\rangle^{\mathrm{T}}$.

\section{Numerical application}

As an illustrative example, the buffeting analysis of a singledegree-of-freedom system is performed with various linearized expressions of an aerodynamic coefficient (as schematically represented in Fig. 3). The considered coefficient is the lift coefficient of the Vasco da Gama bridge (Fig. 2).

\subsection{Probability density function of the wind incidence}

The considered mean wind velocity and wind intensities are respectively $U=10 \mathrm{~m} / \mathrm{s}$ and $I_{u}=I_{w}=5 \%$. The turbulence components are supposed to be perfectly correlated $(\rho=1)$. The proposed method presented in the previous sections, for an effortless determination of the pdf of the wind incidence, is composed of these following steps:

(1) computation of the 36 coefficients $P_{u}, \ldots, P_{w^{3}}$ given in Table 1 for $\rho=1$;

(2) insertion of these coefficients into Eq. (22) for the computation of each statistical characteristic $\left(f=\mu_{i}, \sigma_{i}, \gamma_{3, i}\right.$ or $\left.\gamma_{e, i}\right)$. The resulting values are given in Table 2 (Proposed method);

(3) these statistical characteristics are finally used in Eq. (23) for the establishment of the approached probability density function. This Edgeworth series expansion of the actual probability density function is represented by circles in Fig. 9-a.

In order to validate the procedure, a Monte Carlo simulation is again considered. From a set of $10^{6}$ generated $(u, w)$ couples, a series of wind incidences is built up. The corresponding statistical characteristics are reported in Table 2 and the corresponding normalized histogram is represented in Fig. 9-a. As a complementary comparison, the pdf resulting from the numerical integration in Eq. (7) is also considered. It is represented by dotted lines in Fig. 9-a and the resulting statistical characteristics, obtained by a second numerical integration, are reported in Table 2 (Theoretical).

The very good correspondence between these values and those obtained with the proposed procedure could be reproduced for any $\left(I_{u}, I_{w}\right)$ couple and any correlation coefficient. This validates the proposed method. More generally the good accordance with the Monte Carlo simulation confirms this statement.

\subsection{Fitting of the coefficient}

The lift coefficient of the Vasco da Gama bridge deck is considered (thick line in Fig. 9-b). In this figure the results obtained with the different linearization techniques introduced in Section 2 are represented.

First, the linearization of the aerodynamic coefficient is first realized with the proposed method (circles). The Edgeworth series approximation is used as a weighting function for the least square fitting. This method provides a tight fitting in the vicinity of the origin and deviates from the actual coefficient for larger wind incidences. Secondly, the first order Taylor-McLaurin series expansion (triangles) of the measured aerodynamic coefficient is also considered. It is the tangent to the curve at the origin. The accuracy is very good but in a limited interval around the zero-incidence only. Thirdly, another line (points) is obtained by a least square fitting of the aerodynamic coefficient with uniform weighting along the whole measurement domain $(i \in$ $\left.\left[-10^{\circ},+10^{\circ}\right]\right)$. And finally (crosses), the linearization is performed by simply drawing a line through two considered points $\left(i_{1}=\right.$ $-I_{w}, i_{2}=+I_{w}$ ) as recommended in [12]. Because of the double curvature of the considered lift coefficient, this method provides a linear coefficient similar to the one resulting from the proposed method (at least for positive incidence). This is not observable for any shape of aerodynamic coefficient.

The proposed method is the only one to really account for the actual characteristics of the wind field.

Furthermore, the simple observation of this figure is not sufficient for the determination of the best approximation. The Taylor-McLaurin series approach could be considered to be the worse, but it is actually the best if the wind incidence is very small (note that in this case, the proposed method provides another linearized coefficient and even degenerates into the Taylor series 

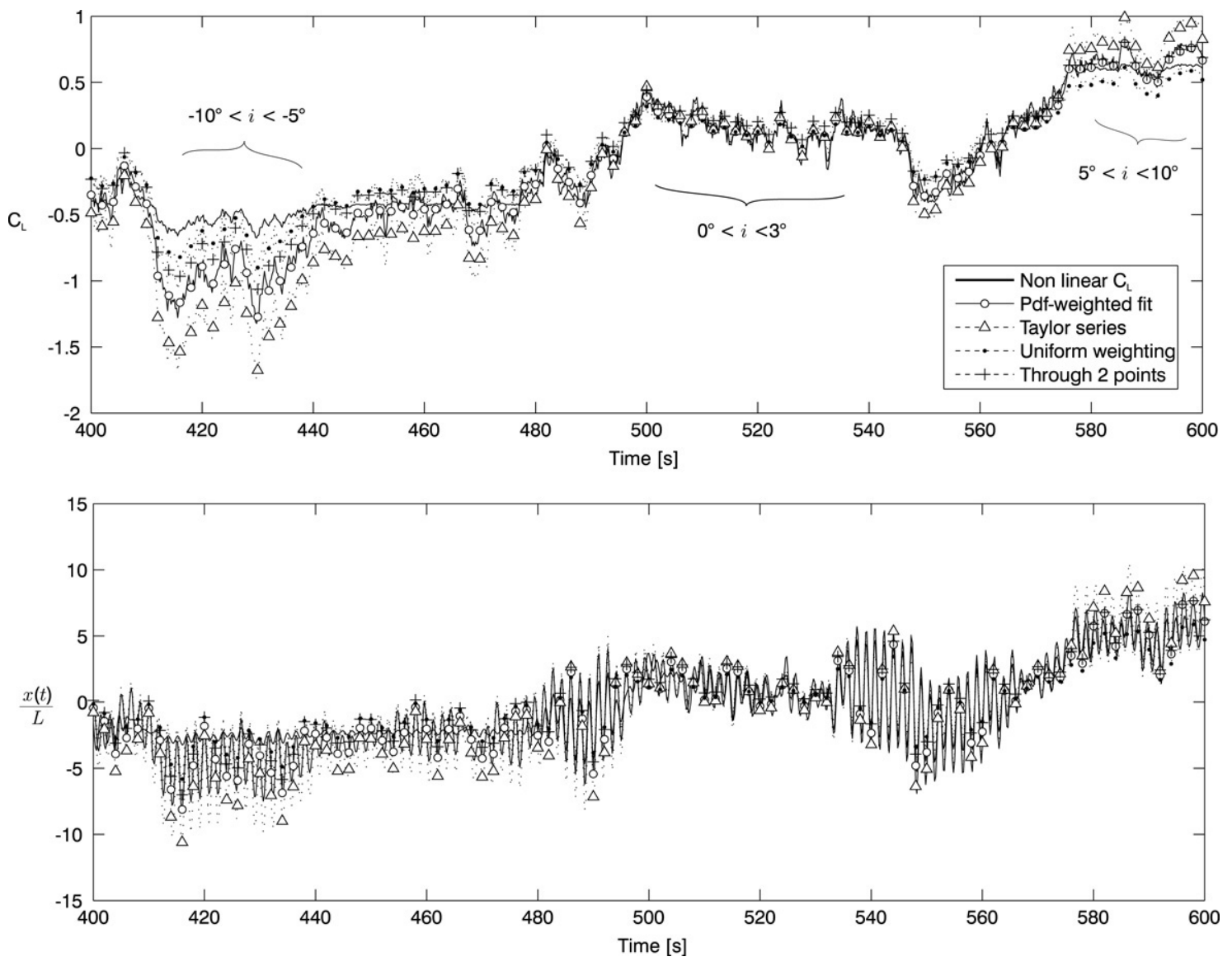

Fig. 10. Time histories of the aerodynamic coefficients and of the corresponding dynamic responses.

approximation for $I_{u}, I_{w} \rightarrow 0$ ). In the context of a buffeting analysis, the best linearization method cannot be awarded without computing the structural response.

\subsection{Time history analysis}

The displacement of the considered single degree of freedom system is governed by:

$\ddot{x}(t)+2 \xi \omega \dot{x}(t)+\omega^{2} x(t)=C[i(t)] \frac{(U+u(t))^{2}+w^{2}(t)}{L}$

where $\omega=4 \mathrm{rad} / \mathrm{s}$ is the circular natural frequency, $\xi=0.01$ is the damping ratio and $L=1 \mathrm{~m}$ is a characteristic length. The stochastic analysis of this SDOF system requires the definition of a frequency content for the loading. The frequency contents of both turbulence components are supposed to be zero-mean Gaussian Orstein-Uhlenbeck processes, i.e. their power spectral densities are given by:

$S_{u}(\omega)=S_{w}(\omega)=\frac{\alpha}{\pi} \frac{U^{2} I_{u}^{2}}{\alpha^{2}+\omega^{2}}$.

This simple random process is sometimes used instead of more accurate analytical formulae, representing more accurately the actual frequency content of the wind [16]. The parameter $\alpha$ acts as a shape factor. In the context of buffeting analysis, it has been shown that $\alpha=0.05 \mathrm{rad} / \mathrm{s}$ is a good representative value [17].

The applied force is a non-linear (quasi-steady) transformation of the wind turbulence components. In order to avoid a complex non-Gaussian analysis in the frequency domain, Eq. (29) is solved in the time domain. $u$ and $w$ are simply generated by an autoregressive filter of a Gaussian white noise process. The considered sampling frequency is $50 \mathrm{~Hz}(\Delta t=0.2 \mathrm{~s})$ and, in order to produce sufficient data for a good statistical estimation up to the fourth order, 150,000 time steps are considered.

After the establishment of the time history of the wind incidence, the time histories of aerodynamic coefficients are obtained with each considered linearization technique. Fig. 10 represents a window of the whole generated series. When the wind incidence is close to the mean value $(t \simeq 520 \mathrm{~s})$, the coincidence between all aerodynamic coefficients is good (see Fig. 9-b). As soon as the wind incidence takes extreme values, the discrepancies are more noticeable. This results in a significant difference between the actual coefficient (solid thick line) and the linearized expressions.

The response of the single degree of freedom system is also reported in Fig. 10. The time histories of the structural displacement indicate a compelling dynamic counterpart. Actually the ratio between the background and the resonant components is around $2: 1$, which is a common value usually encountered in the context of cable-stayed bridges [18].

Numerical values of the mean, standard deviation and extreme values of $x / L$ are given in Table 3 and illustrated in Fig. 11. The results obtained with each linear aerodynamic coefficient are compared to the rigorous one and reported as an error. Although the considered turbulence intensities are small $\left(I_{u}=I_{w}=5 \%\right)$, the Taylor series expansion provides excessively overestimated standard deviation and extreme values (up to $43 \%$ ). On the contrary, a least square method with uniform weighting supplies drastically unsafe results (up to $-30 \%$ ). In this application, the simple linearization technique consisting in using a line passing through two chosen points gives very good results, even the best extreme values. The 
Table 3

Statistical characteristics of the response obtained with several linearization techniques

\begin{tabular}{|c|c|c|c|c|c|c|c|c|c|}
\hline \multirow[t]{2}{*}{ Statistics of $\frac{x}{L}$} & \multirow[t]{2}{*}{$\mu$} & \multicolumn{2}{|l|}{$\sigma$} & \multicolumn{3}{|l|}{$\max$} & \multicolumn{3}{|l|}{$\min$} \\
\hline & & & (err \%) & & (err \%) & peak & & (err \%) & peak \\
\hline Non-linear coefficient & -0.040 & 2.63 & - & 10.37 & - & 3.95 & -8.60 & - & 3.25 \\
\hline Proposed method & -0.067 & 2.49 & $(-5.5)$ & 10.76 & $(3.8)$ & 4.35 & -9.43 & $(9.7)$ & 3.76 \\
\hline Taylor series & -0.299 & 3.18 & $(20.9)$ & 13.55 & $(30.7)$ & 4.35 & -12.28 & $(42.9)$ & 3.76 \\
\hline Uniform weighting & 0.146 & 1.85 & $(-29.8)$ & 8.18 & $(-21.1)$ & 4.35 & -6.80 & $(-20.8)$ & 3.76 \\
\hline Through 2 points & 0.476 & 2.32 & $(-12.0)$ & 10.55 & $(1.8)$ & 4.35 & -8.24 & $(-4.2)$ & 3.76 \\
\hline
\end{tabular}

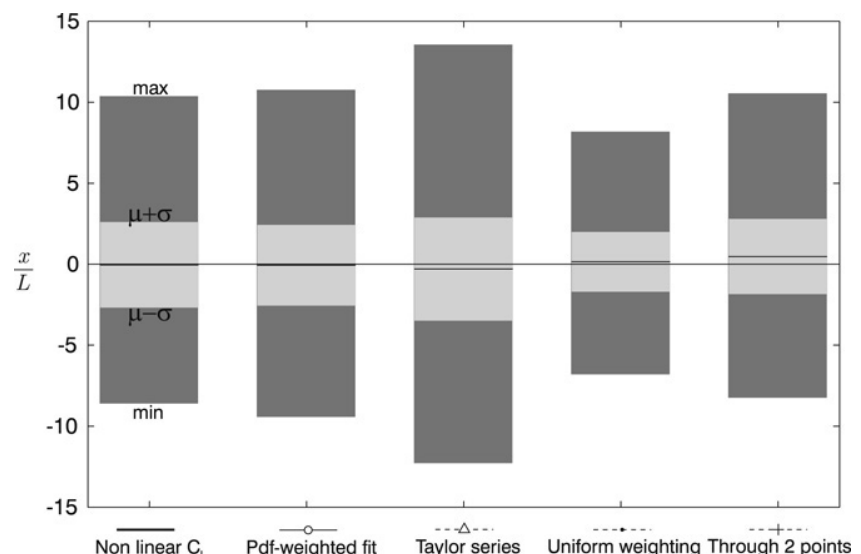

Fig. 11. Statistics of the structural response for different linearization techniques of the aerodynamic coefficient (mean plus/minus standard deviation, extreme values). The first result (Non-linear $C_{L}$ ) is the reference result to which all four other methods are compared.

conclusion can not be generalized, however, simply because it does not account for the shape of the aerodynamic coefficient between both chosen points. Finally, the proposed method provides the smallest error on the estimation of the standard deviation, which was expected as an application of the stochastic linearization theory.

The discrepancies between the exact extreme values and those resulting from the linear models are closely related to the nonGaussianity of the response. As a persuasive argument, the peak factors computed by:

$g_{\max }=\frac{\max -\mu}{\sigma} ; \quad g_{\min }=\frac{\min -\mu}{\sigma}$

are identical for all linearized models, but different than the exact one. In the computations with linearized coefficients, the nonGaussianity comes from the squared velocity only, whereas it has a second origin when the non-linearity of the aerodynamic coefficient is considered. Since the actual peak factors are larger than those obtained with the linear models, it is expected that the extreme values resulting from the linearized computation provide also larger extreme values. This is well the case for the proposed method.

\section{Conclusions}

The linearization of aerodynamic coefficients resulting from wind tunnel experiments has to be performed carefully. In the context of a buffeting analysis, the time variation of the wind incidence must be considered. Based on the stochastic linearization technique, it is proposed to perform the linearization by the least square method, with a weighting function proportional to the probability density function of the wind incidence.
The problem of the computation of the probability density function of the wind incidence in a 2-D wind flow with Gaussian turbulence can be considered to be solved. Thanks to numerical developments, it is expressed by simple functions of $I_{u}, I_{w}$ and $\rho$ (Eq. (23), Eq. (22) and Table 1).

The combination of these relations and the least square fitting method with a non-uniform weighting (Section 5) results in the proposed method for the replacement of measured aerodynamic coefficients by polynomial approximations.

A numerical application has shown the optimality of the proposed method for buffeting analysis and has illustrated that other systematic linearization techniques can lead to significantly safe or unsafe errors on the estimation of the structural response. Because it involves precisely the statistical characteristics of the wind field, the proposed method is accurate, and the proposed relations offer a simple way to provide reliable estimations of the structural response.

\section{References}

[1] Larsen A, Walther JH. Aeroelastic analysis of bridge girder sections based on discrete vortex simulations. Journal of Wind Engineering and Industrial Aerodynamics 1997;67-68:253-65.

[2] Chen X, Kareem A. Advances in modelling of aerodynamic forces on bridge decks. Journal of Engineering Mechanics 2002;128(11):1193-250.

[3] Dyrbye C, Hansen SO. Wind loads on structures. New York: John Wiley \& Sons; 1997.

[4] Holmes JD. Wind loading on structures. London: SpronPress; 2001.

[5] Singh L, Jones NP, Scanlan RH, Lorendeaux O. Identification of lateral flutter derivatives of bridge decks. Journal of Wind Engineering and Industrial Aerodynamics 1996;60(1):81-9.

[6] Gu M, Qin XR. Direct identification of flutter derivatives and aerodynamic admittances of bridge decks. Engineering Structures 2004;26(14):2161-72.

[7] Simiu E, Scanlan RH. Wind effects on structures. New York: John Wiley \& Sons; 1996.

[8] Di Paola M, Falsone G, Pirrotta A. Stochastic response analysis of nonlinear systems under gaussian inputs. Probabilistic Engineering Mechanics 1992;7: 15-21.

[9] Grigoriu M. Response of linear systems to quadratic gaussian excitation. Journal of Engineering Mechanics ASCE 1986;112(6):729-44.

[10] Denoël V, Degée H. Influence of the non-linearity of the aerodynamic coefficients on the skewness of the buffeting drag force. International Journal of Wind and Structures 2006;9(6):457-71.

[11] Roberts JB, Spanos PD. Random vibration and statistical linearization. Chichester: Wiley; 1991.

[12] de Ville de Goyet V. Analyse spectrale du comportement au vent turbulent du Viaduc de Millau, linéarisation des coefficients aérodynamiques. Technical Report, BEG design office, No. NT-030, 2005.

[13] Zhou Y, Kijewski T, Kareem A. Along-wind load effects on tall buildings: Comparative study of major international codes and standards. Journal of Structural Engineering 2002;128(6):788-96.

[14] Cramér H. Mathematical methods of statistics. Princeton: Princeton University Press; 1945.

[15] Lawson C, Hanson R. Solving least squares problems. Englewood Cliffs: Prentice-Hall; 1974.

[16] Floris C. Equivalent gaussian process in stochastic dynamics with application to along-wind response of structures. International Journal of Non-Linear Mechanics 1996;31(5):779-94.

[17] Denoël V. Application of stochastic analysis methods to the study of wind effects on civil engineering structures. Ph.D. thesis. Belgium: University of Liège; 2005 [in French].

[18] Denoël V, Degée H, de Ville de Goyet V. Importance of modal cross-correlations on wind loaded structures. In: Proceedings of the 3rd international conference on bridge maintenance, safety and management, Porto, IABMAS 06, 2006. 